\title{
Primeiro registro de Phanoxyla hystrix (R. Felder, [1874]) (Lepidoptera, Sphingidae) para o Estado de Rondônia, Brasil
}

Josilândia Silva DUARTE ${ }^{1}$, Catarina da Silva MOTTA², Gilcélia Melo LOURIDO³

\section{RESUMO}

Um macho de Phanoxyla hystrix (R. Felder, [1874]) foi coletado em Porto Velho, representando o primeiro registro para o estado de Rondônia, Brasil.

PaLAVRAS-CHAVE: Lepidoptera, Sphingidae, Phanoxyla hystrix, Amazônia Brasileira.

\section{First record of Phanoxyla hystrix (R. Felder, [1874]) (Lepidoptera, Sphingi- dae) in the Rondonia State, Brazil}

\section{ABSTRACT}

A male specimen of Phanoxyla hystrix (R. Felder, [1874]) was collected in Porto Velho representing the first record from Rondonia State, Brazil.

KEYWORDS: Lepidoptera, Sphingidae, Phanoxyla hystrix, Brazilian Amazon.

\footnotetext{
Laboratório de Entomologia, Universidade de Rondônia - UNIR. BR 364, km 9,5, Zona Rural, Campus José Ribeiro Filho CEP 78900-000 Porto Velho, Rondônia, Brasil. e-mail: josilandia_duarte@yahoo.com.br

2 Coordenação de Pesquisas em Entomologia, Instituto Nacional de Pesquisas da Amazônia - INPA. Caixa Postal 478, 69011-970 Manaus, Amazonas, Brasil. e-mail: motta@inpa.gov.br telefone: 9236433221

${ }^{3}$ Divisão do Curso em Entomologia, INPA. e-mail: lourido@inpa.gov.br
} 
Phanoxyla hystrix (R. Felder, [1874]) (Lepidoptera, Sphingidae) foi descrita inicialmente no gênero Chaerocampa Duponchel, 1835 por Felder (1874) com base num exemplar macho coletado por Bates no Rio Amazonas, tipo ex Coll Felder, e depositado no Tring Museum, atualmente no British Museum (Natural History) - BM(NH)

Rothschild e Jordan (1903) em uma revisão dos Sphingidae, ao criarem o gênero Phanoxyla para esta espécie, mencionaram outro exemplar macho depositado na coleção Charles Oberthür proveniente do município São Paulo de Olivença, estado do Amazonas, coletado em vi.vii.1883 por M. de Mathan. Atualmente esse exemplar encontra-se também depositado no BMNH.

Schreiber (1978) relata a ocorrência desta espécie para o México, Venezuela, Colombia, Equador, Peru, Argentina e norte do Brasil. De acordo com o autor, todos os registros, salvo Equador e o norte do Brasil, são baseados em apenas um espécime.

Os registros geográficos de $P$. hystrix são escassos, além dos trabalhos de Felder [(1874)], Rothschild e Jordan (1903) e Schreiber (1978) são conhecidos apenas os de Boisduval (I874),
Butler (1877), Kirby (1892) e Kitching e Cadiou (2000), tratando de breves descrições e sinonímias para essa espécie.

No CATE (Creating a Taxonomic e-Science) são ilustradas as fotos de um macho proveniente de Mangosissa (erro gráfico); "Magosiza", Equador, e da genitália de outro macho, também do Equador, porém de Macas, além da genitália de uma fêmea proveniente de Contamana, Rio Ucayali, Peru.

No Museu Paraense Emílio Goeldi encontra-se depositado outro macho, constando na etiqueta apenas 'Óbidos', município do estado do Pará.

Este é o primeiro registro de $P$. hystrix para o estado de Rondônia, coletado em Porto Velho. O espécime macho , fotografado em vistas dorsal e ventral (Figs. 1 e 2), foi coletado manualmente, na parede branca, externa do Laboratório de Entomologia, Universidade Federal de Rondônia, durante o dia, em 06.x.2004 por Duarte, J.S., e está depositado na coleção de Invertebrados do Instituto Nacional de Pesquisas da Amazônia - INPA, sob número de registro 69787.

Imaturos e plantas hospedeiras dessa espécie são desconhecidas.

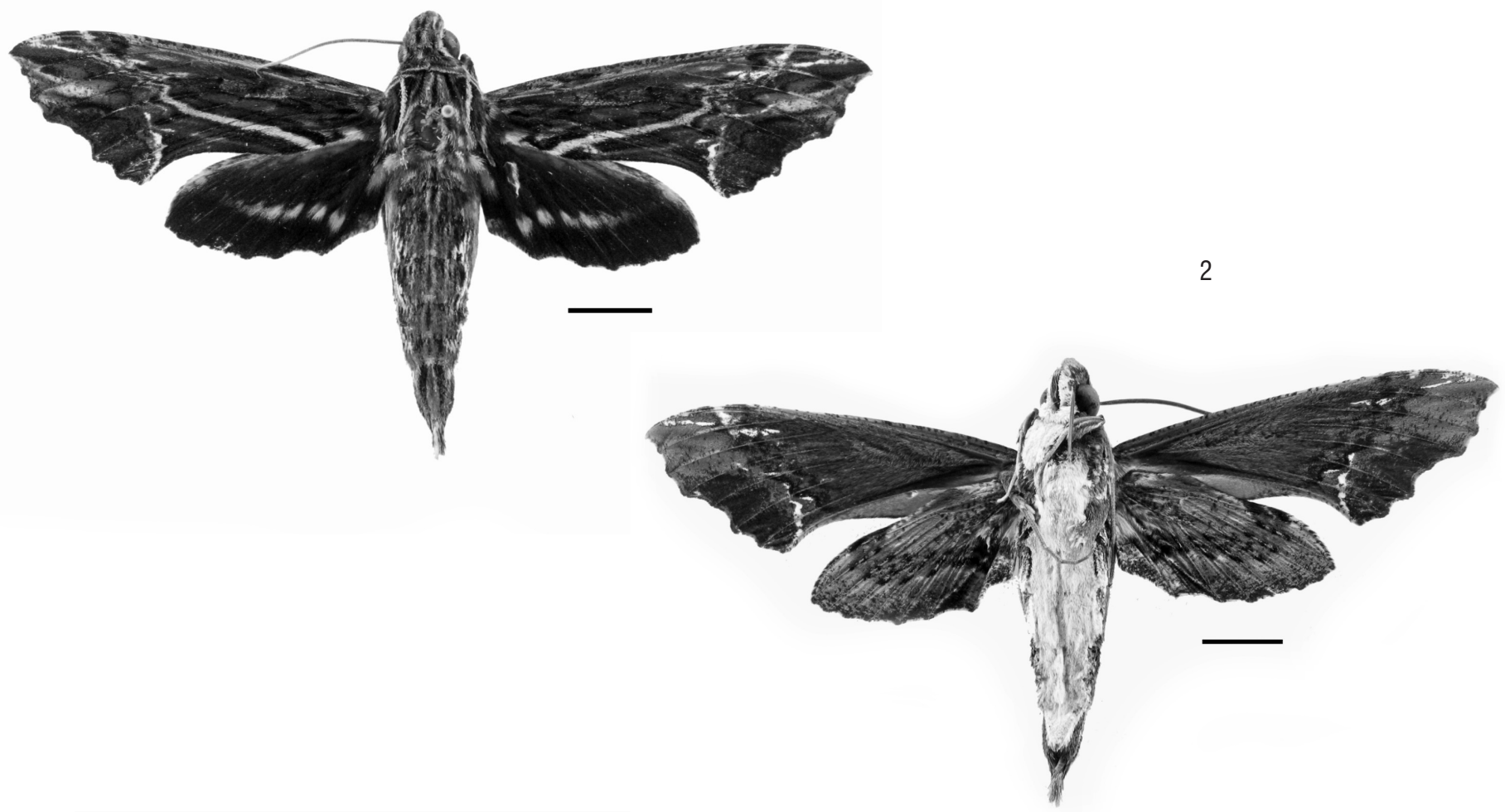

Figuras 1-2 - Phanoxyla hystrix: (1) vista dorsal; (2) vista ventral. Escala 10mm. 


\section{AGRADECIMENTOS}

As autoras agradecem ao Roger Hutchings pela ajuda com o abstract.

\section{BIBLIOGRAFIA CITADA}

Boisduval, A. 1874. Tome premier. Sphingides, Sesiides, Castnides. In: J.B.A. Boisduval; Guénée A. (Eds.). Histoire naturelle des Insectes. Spécies general des Lépidoptères. Paris, Librairie Encyclopédique de Roret. p. 4-568.

Butler, A.G. 1877. Revision of the Heterocerous Lepidoptera of the family Sphingidae. Transactions of the Zoological Society of London, 9: 511-644, 5 pls.

CATE 2008 (Creating a Taxonomic e-Science). Disponível em: http://www.cate-project.org. Acesso em: 20/06/2008.

Felder, R. 1874. Lepidoptera. Heft IV. Atlas der Heterocera Sphingida-Noctuida. In: Felder, R.; Rogenhofer A.F. (Eds.). Reise der österreichischen Fregatte Novara um die Erde in den Jahren 1857, 1858, 1859, unter den befehlen des Commodore B. Von Willerstorf-Urbair, Zoologisher Theil, Band 2 (Abtheilung 2). Vienna, Der kaiserlich-königlichen Hof- und Staatsdruckerei. p. 1-10. Plates 75-107.
Kirby, W.F., 1892. A Synonymic Catalogue of Lepidoptera Heterocera (Moths). Vol. 1. Sphinges and Bombyces. London, Gurney \& Jackson. 951pp.

Kitching, I.J.; Cadiou J.M.. 2000. Hawkmoths of the World: An annotated and illustrated revisionary checklist (Lepidoptera: Sphingidae). Ithaca and London, Cornell University Press. viii+227pp.

Rothschild, W.; Jordan K. 1903. A Revision of the Lepidopterous family Sphingidae. Novitates Zoologicae, 9 (suppl.): 1-972, 67 pls.

Schreiber, H. 1978. Dispersal Center of Sphingidae (Lepidoptera) in the Neotropical Region. Biogeographica, 10: 1-195.

Recebido em 19/09/2008

Aceito em 03/11/2008 
